\title{
Psoríase pustulosa palmoplantar tratada com etanercepte: relato de caso
}

\section{Palmoplantar pustular psoriasis treated with etanercept}

\section{Fernanda de Sousa Ferrara ${ }^{1}$, Camila Oliveira Alvarenga ${ }^{2}$, Maria do Rosário Vidigal ${ }^{3}$, Antônio José Tebcherani ${ }^{4}$, Ana Paula Galli Sanchez ${ }^{5}$} Ferrara FS, Alvarenga CO, Vidigal MR, Tebcherani AJ, Sanchez APG. Psoríase pustulosa palmoplantar tratada com etanercepte: relato
de caso / Palmoplantar pustular psoriasis treated with etanercept. Rev Med (São Paulo). 2011 jul.-set.;90(3):128-32.

RESUMO: Contexto: A psoríase pustulosa palmoplantar (PPPP) é uma das apresentações clínicas da psoríase, muitas vezes de difícil tratamento, podendo-se utilizar diversos medicamentos tópicos e sistêmicos. O uso dos anti-TNF $\alpha$ no tratamento das formas pustulosas de psoríase é controverso, visto que a resposta clínica é variável, além desta classe de medicação biológica poder desencadear psoríase pustulosa. Descrição do Caso: Doente feminina, 60 anos, branca, com diagnóstico de PPPP há 12 anos e artrite psoriásica há seis anos. Antecedentes pessoais relevantes: hipertensão arterial sistêmica, hipertrigliceridemia e obesidade. Apresentou refratariedade tanto aos tratamentos tópicos instituídos (corticóides e emolientes) quanto aos sistêmicos (metotrexate, dapsona, colchinina e acitretina), tendo evoluído com excelente resposta terapêutica com etanercepte (administrado semanalmente, por via subcutânea, na dose de $50 \mathrm{mg}$ ). Discussão: Os anti-TNF $\alpha$ são eficazes no tratamento da psoríase em placas moderada a grave. Mas, o tratamento da PPPP com anti-TNFa não é classicamente preconizado. Segundo a literatura a resposta terapêutica com este tipo de medicamento é variável na PPPP. Além disso, os anti-TNF $\alpha$ podem desencadear quadro de pustulose palmoplantar. No entanto, há relatos de sucesso terapêutico com anti-TNF $\alpha$ em casos refratários de PPPP. Em função das comorbidades da paciente e após terem sido esgotadas as possibilidades terapêuticas clássicas, optamos pelo uso do etanercepte, que se mostrou eficaz. A doente iniciou tratamento em abril de 2008 e mantém o uso do etanercepte até a presente data, estando em remissão da doença. Conclusão: Destacamos a possibilidade do uso de anti-TNF $\alpha$ em paciente com PPPP refratária ao tratamento convencional. No nosso caso, o etanercepte mostrou-se eficaz e seguro, não tendo a doente apresentado nenhum efeito adverso grave.

DESCRITORES: Psoríase; Qualidade de vida; Dermatopatias,;Artrite psoriásica; Fator de necrose tumoral alfa.
ABSTRACT: BACKGROUND: Palmoplantar pustular psoriasis (PPPP) is one of the clinical presentations of psoriasis, often difficult to treat, can be used several topical and systemic. The use of anti-tumor necrosis factor alpha (anti-TNF $\alpha$ ) in the treatment of pustular forms of psoriasis is controversial, with a variable clinical response, outside this class of biological medication may trigger pustular psoriasis. CASE REPORT: Patient 60 , female, white, with a diagnosis of PPPP for 12 years and psoriatic arthritis for 6 years. Relevant personal history: hypertension, hypertriglyceridemia and obesity. Presented refractory to topical treatments (corticosteroids and emollients) and systemic (methotrexate, dapsone, colchicine and acitretin), having evolved with excellent response to treatment with etanercept (administered weekly by subcutaneous injection at a dose of $50 \mathrm{mg}$ ). DISCUSSION: The anti-TNF $\alpha$ are effective in the treatment of psoriasis in moderate to severe plaque. But, in the treatment of PPPP is not classically recommended. According to the literature, the therapeutic response with this type of drug is variable in PPPP. In addition, anti-TNF $\alpha$ may trigger clinical of palmoplantar pustulosis. However, there are reports of therapeutic success with anti-TNF $\alpha$ in refractory cases of PPPP. Due to patient's comorbidities, and after having exhausted the possibilities classical therapies, we chose the use of etanercept, which proved effective. The patient began treatment in April 2008 and keeps the use of etanercept until the present date, being in remission. CONCLUSION: We emphasize the possibility of using anti-TNF $\alpha$ in patients with PPPP refractory to conventional treatment. In our case, etanercept was effective and safe, not having the patient presented any serious adverse events.

KEYWORDS: Psoriasis; Quality of life; Skin diseases; Arthritis, psoriatic; Tumor necrosis factor-alpha.

Pesquisa desenvolvida no Serviço de Dermatologia do Complexo Hospitalar Padre Bento de Guarulhos, SP.

1. Médica colaboradora do Serviço de Dermatologia do Complexo Hospitalar Padre Bento de Guarulhos.

2. Médica colaboradora do Serviço de Dermatologia do Complexo Hospitalar Padre Bento de Guarulhos.

3. Diretora Técnica de Serviço de Saúde do Núcleo de Ambulatório de Dermatologia do Complexo Hospitalar Padre Bento de Guarulhos; médica Dermatologista e Hansenóloga do Serviço de Dermatologia do Complexo Hospitalar Padre Bento de Guarulhos.

4. Mestre em Patologia pela UNIFESP, Patologista do Serviço de Dermatologia do Complexo Hospitalar Padre Bento de Guarulhos.

5. Mestre em Ciências pela Faculdade de Medicina da Universidade de São Paulo; médica dermatologista do Serviço de Dermatologia do Complexo Hospitalar Padre Bento de Guarulhos.

Endereço para correspondência: Fernanda S. Ferrara. Rua Atalanta, 125 - Bairro: Jardim São Paulo, São Paulo, SP, CEP: $02040-020$. E-mail: ferferrara@gmail.com 


\section{INTRODUÇÃO}

A psoríase é dermatose inflamatória crônica que acomete cerca de $1 \%$ da população brasileira ${ }^{1}$. Sua etiopatogenia envolve fatores genéticos, imunológicos e ambientais ${ }^{2,3}$. Caracteriza-se por lesões cutâneas localizadas ou disseminadas, associadas ou não a quadro articular e a outras comorbidades (doenças auto-imunes, hipertensão arterial, diabetes, hiperlipidemia, obesidade, depressão) ${ }^{2-4}$.

A forma clínica mais comum da psoríase é a psoríase em placas (lesões eritêmato-escamosas), que ocorre em cerca de $80 \%$ dos pacientes ${ }^{2}$. A psoríase pustulosa palmoplantar (PPPP) é variante clínica incomum da psoríase, de difícil tratamento e que compromete de forma significativa a qualidade de vida dos doentes ${ }^{5}$. Acomete mais freqüentemente mulheres e tabagistas ${ }^{6}$, entre 40 a 60 anos $^{7}$. Caracteriza-se pela erupção de pústulas estéreis, recorrentes, associadas à lesões eritêmatoescamosas. Ocorre geralmente de forma bilateral e simétrica nas regiões das palmas e plantas ${ }^{6}$. Quando a erupção das pústulas acontece no leito ungueal (gerando distrofia ungueal) e nas extremidades distais dos dedos, temos a Acrodermatite de Hallopeau. O diagnóstico da PPPP pode ser corroborado pelo anatomopatológico, destacando-se o acúmulo de neutrófilos na epiderme (pústulas espongiformes de Kogoj e microabscessos de Munro) ${ }^{6}$.

O tratamento da PPPP é difícil, pois a maioria dos casos é refratária aos tratamentos tópicos e sistêmicos clássicos, que incluem: corticóides tópicos, fototerapia, metotrexate, ciclosporina e acitretina. O uso dos anti-TNF $\alpha$ no tratamento da PPPP é controverso, visto que este tipo de medicação pode desencadear quadros de pustuloses palmoplantares ${ }^{8}$.

\section{DESCRIÇÃO DO CASO}

Doente feminina, 60 anos, branca, com diagnóstico de PPPP há 12 anos e artrite psoriásica há seis anos. Antecedentes pessoais relevantes: hipertensão arterial sistêmica, obesidade e hipertrigliceridemia. Procurou o Serviço de Dermatologia do Complexo Hospitalar Padre Bento de Guarulhos, SP, em 2002 após tratamento prévio com metotrexate e fototerapia sem melhora.

Apresentava lesões pustulosas palmoplantares, além de acometimento das unhas e das extremidades dos dedos das mãos. Referia limitação importante do uso das mãos bem como da deambulação. O exame anatomopatológico confirmou o diagnóstico de psoríase (Figura 1). As tentativas de controle do quadro com dapsona e colchicina foram insatisfatórias, visto que o uso dessas medicações não pôde ser mantido em função de efeitos adversos (respectivamente, erupção medicamentosa e intolerância gastrointestinal). Iniciado em 2004 tratamento com acitretina (25 mg/dia), com melhora parcial do quadro. Porém, na vigência do tratamento regular com acitretina, a paciente apresentava surtos de piora da PPPP e sua dislipidemia não mostrava controle satisfatório com as medicações instituídas pela endocrinologia. Além disso, desenvolveu artrite psoriásica.

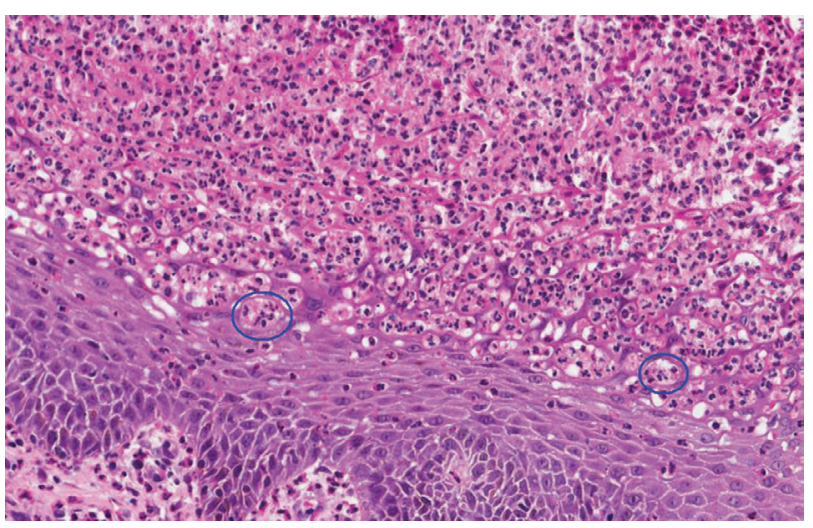

Figura 1. Intensa migração neutrofílica na superfície da epiderme, notando-se ausência da camada granulosa e as pústulas de Kogoj (círculos azuis). HE. 200x

Em função da falta de resposta prévia ao metotrexate e das comorbidades (hipertensão arterial, obesidade e dislipidemia), decidimos por iniciar etanercepte com o objetivo de controlar o quadro cutâneo e articular da psoríase. Foram realizados exames para o início do tratamento com anti-TNF $\alpha$ dentre eles: hemograma, função renal e hepática (normais), sorologias para hepatite B e C (negativas), anti-HIV (negativo), intradermorreação à tuberculina (negativa) e radiografia do tórax (sem alterações).

A doente iniciou em abril de 2008 tratamento com etanercepte (50 mg semanal por via subcutânea). No começo, usou o etanercepte em associação com a acitretina ( $25 \mathrm{mg} / \mathrm{dia})$ (Figuras 2,3 e 4). A dose da acitretina foi progressivamente diminuída, sendo suspensa em julho de 2008. Mantida a monoterapia com o etanercepte até a presente data (Figuras 5,6 e 7). A melhora clínica com o etanercepte foi rápida, sendo evidente a partir da oitava semana. O medicamento foi interrompido apenas para a realização de herniorrafia abdominal em 2010 e durante dois episódios de infecções de vias aéreas superiores. A paciente apresentou também melhora significativa da sua qualidade de vida (DLQI Dermatoly Life Quality Index - inicial de 22 e atual 
igual a três). O uso do etanercepte permitiu o controle do quadro cutâneo e articular. Eventualmente, refere surgimento de algumas pústulas isoladas nas mãos, que desaparecem com a aplicação de corticóide de alta potência (betametasona creme) e emolientes. DISCUSSÃO

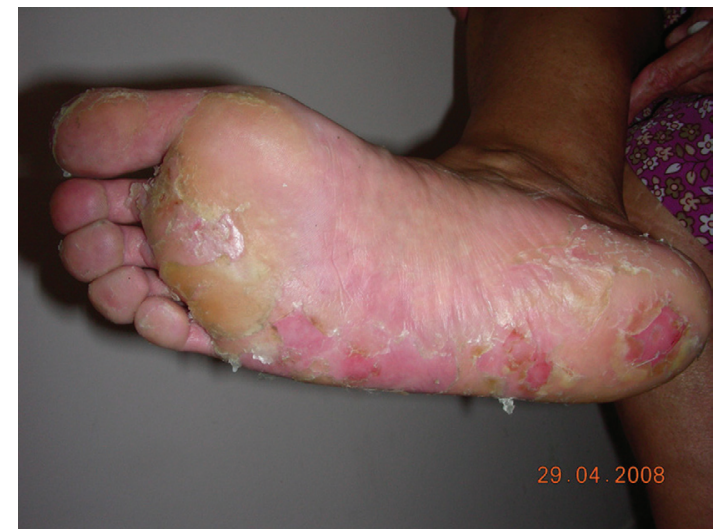

Figura 2. Placas eritêmato-descamativas e algumas pústulas na planta do pé direito antes do tratamento com etanercepte e em uso de acitretina (abril de 2008)

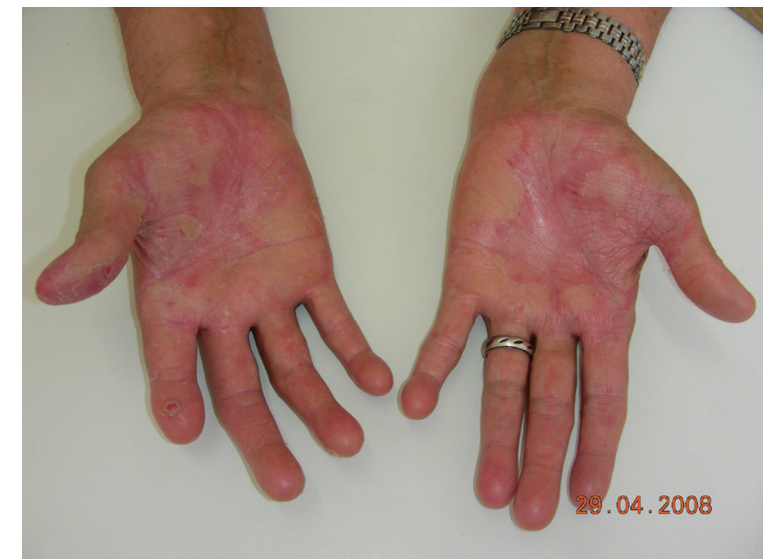

Figura 3. Placas eritêmato-descamativas nas palmas em uso de acitretina (abril de 2008)

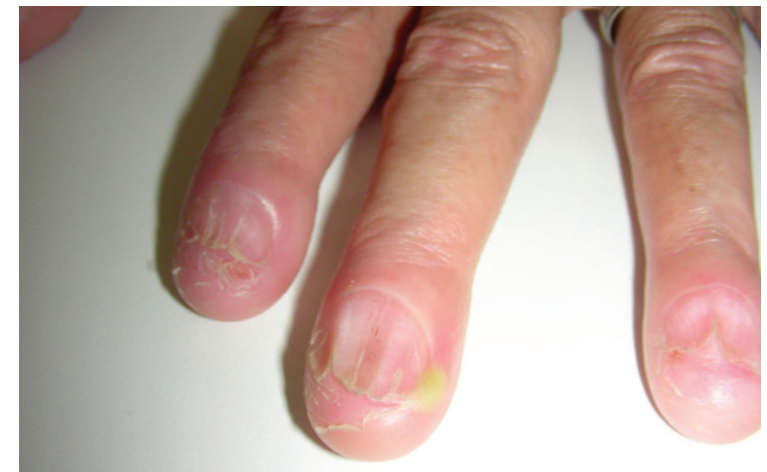

29.04 .2008

Figura 4. Distrofia ungueal e pústula no terceiro quirodáctilo esquerdo em uso de acitretina (abril de 2008)

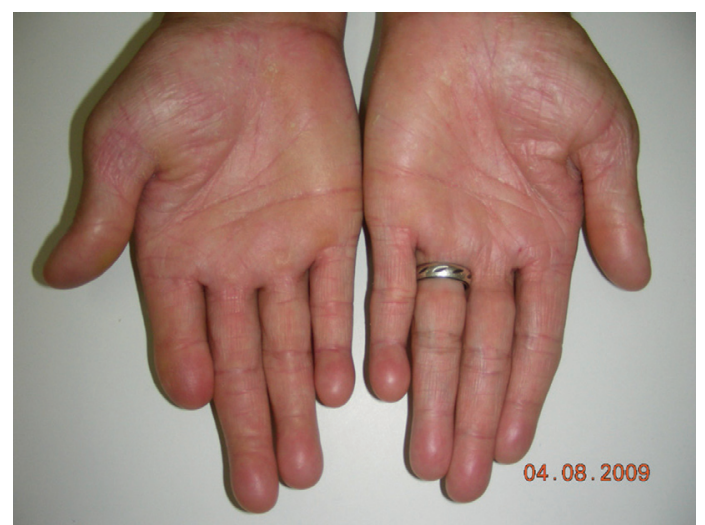

Figura 5. Leve eritema e descamação nas palmas em monoterapia com etanercepte (agosto de 2009)

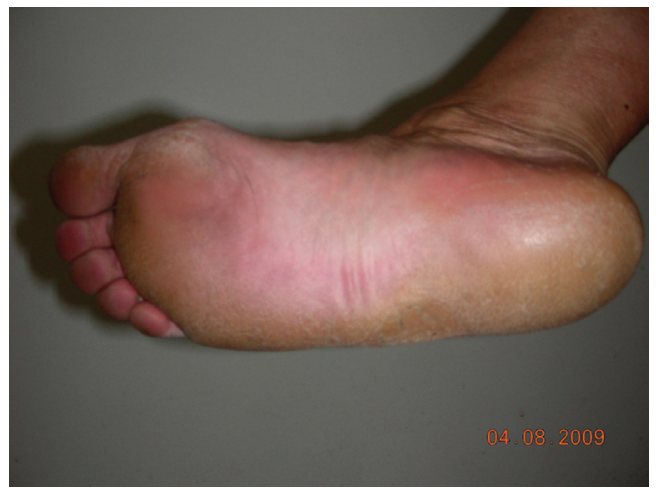

Figura 6. Ausência de lesões na planta do pé direito em monoterapia com etanercepte (agosto de 2009)

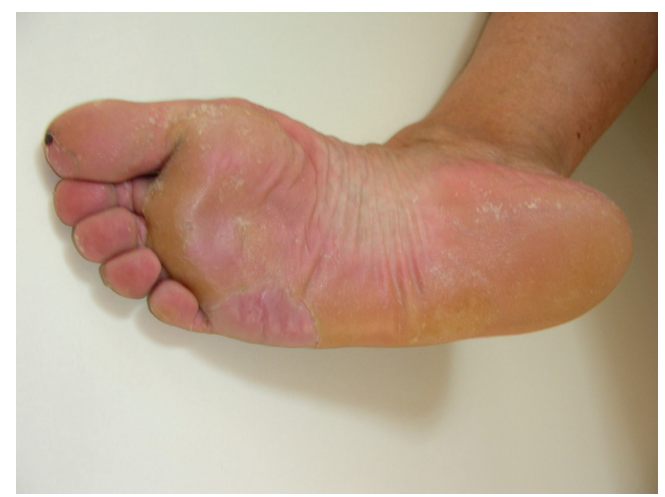

Figura 7. Áreas de descamação e hiperceratose na planta do pé direito (2011). Ausência de pústulas

A psoríase é atualmente considerada uma doença inflamatória imunomediada pelos linfócitos T (LT) CD4+ do tipo 1 e do tipo $17^{9}$. Os LTCD4+ do tipo 17 são grandes produtores de IL-17, TNF $\alpha$ e IL-6, citocinas envolvidas na patogênese da aterosclerose, o que justifica nos doentes com psoríase grave o maior risco de desenvolvimento da síndrome metabólica e de doença cardiovascular ${ }^{4,9}$.

O TNF $\alpha$ é uma das principais citocinas 
envolvidas na imunopatogênese da psoríase ${ }^{9}$, sendo o uso dos anti-TNF $\alpha$ comprovadamente eficaz no tratamento da psoríase em placas (moderada a grave) e na artrite psoriásica ${ }^{1,10}$.

No Brasil os anti-TNF $\alpha$ aprovados para o tratamento da psoríase incluem dois tipos':

1) Anticorpos monoclonais: Infliximabe (Imunoglobulina G quimérica) e Adalimumabe (Imunoglobulina G humana);

2) Proteína de fusão humana: Etanercepte (formado pelo receptor do TNF acoplado à fração cristalizável de uma IgG).

No tratamento da PPPP os anti-TNF $\alpha$ não são classicamente preconizados, podendo inclusive desencadear quadros de pustuloses palmo-plantares ${ }^{11}$. No entanto, vem sendo relatada a eficácia dos anti-TNF $\alpha$ no tratamento de doentes com quadros de PPPP refratários aos tratamentos tópicos e sistêmicos convencionais ${ }^{12-15}$.

Na nossa doente, em função da refratariedade aos tratamentos clássicos, das comorbidades e do surgimento da artrite psoriásica, optamos pelo tratamento com etanercepte. Outra razão importante para o início da medicação biológica foi o acentuado comprometimento da sua qualidade de vida (DLQI igual a 22). A propósito, o DLQI Dermatoly Life Quality Index - é um questionário com 10 questões, cuja pontuação varia de zero a $30^{16}$. $O$ comprometimento da qualidade de vida é considerado grave na psoríase quando o DLQI é maior que $10 \mathrm{e}$ leve se variar de dois a cinco ${ }^{17}$.

Antes do início do etanercepte foram realizados os exames gerais preconizados ${ }^{1,2,10}$ acima relatados, destacando-se a necessidade da realização do screening para tuberculose latente ( $R X$ de tórax e intradermorreação à tuberculina). Os exames laboratoriais de controles (hemograma, função hepática e renal) foram feitos após um mês do início da medicação e repetidos a cada três meses, mantendo-se dentro da normalidade. Repetimos anualmente as sorologias e a intradermorreação à tuberculina (que se mantêm negativas) ${ }^{10}$.

\section{REFERÊNCIAS}

1. Sociedade Brasileira de Dermatologia. Consenso Brasileiro de Psoríase - 2009. Rio de Janeiro: SBD; 2009.

2. Menter A, Gottlieb A, Steven R, Feldman SR, Van Voorhees AS, Leonardi CL, et al. Guidelines of care for the management of psoriasis and psoriatic arthritis. Section 1. Overview of psoriasis and guidelines of care for the treatment of psoriasis with biologics. J Am Acad Dermatol. 2008;578:826-50.
Éfundamental que seja realizado regularmente o seguimento clínico e laboratorial dos doentes em tratamento com anti-TNF $\alpha$, atentando-se para o maior risco de infecções e para a monitorização do eventual surgimento de neoplasias nesses doentes, já que esses medicamentos modulam o sistema imunológico ${ }^{18}$. Durante os quatro anos de tratamento com etanercepte nossa paciente apresentou como efeito adverso apenas dois episódios de infecções das vias respiratórias altas, que são o tipo de infecção mais comumente descrito com o uso dos anti-TNF ${ }^{10}$. A doente realiza sozinha a aplicação da injeção subcutânea semanal do etanercepte, estando orientada a não realizar a aplicação se febre, ou seja, na vigência de infecção (tendo suspendido a medicação quando iniciou os sintomais gripais). Vale ressaltar que os anti-TNF $\alpha$ podem predispor os doentes a infecções graves, mas este é um efeito adverso raro $^{19}$.

Na nossa doente, a medicação foi também interrompida para a realização de cirurgia eletiva, sendo posteriormente reintroduzida sem comprometimento da sua eficácia. Dentre os anti-TNF $\alpha$, o etanercepte pode ser empregado de forma intermitente no tratamento da psoríase em placas, em geral sem comprometimento da eficácia terapêutica ${ }^{20-23}$, como observamos neste caso de PPPP.

Por fim, ressaltamos a benefício da medicação na melhora da qualidade de vida da paciente, que voltou a executar satisfatoriamente suas atividades habituais.

\section{CONCLUSÃO}

Destacamos a possibilidade do uso de anti-TNF $\alpha$ em pacientes com PPPP refratária aos tratamentos clássicos. No caso apresentado o etanercepte vem se mostrando eficaz e seguro a longo prazo no tratamento da PPPP e da artrite psoriásica.
3. Nestle FO, Kaplan DH, Barker J. Psoriasis. Mechanisms of disease. N Engl J Med. 2009;361:496-509.

4. Kourosh AS, Miner A, Menter A. Psoriasis as a marker of underlyning systemic disease. Skin Ther Lett. 2008;13:1-5.

5. Mrowietz $U$, Van de Kerkhof P. Management of palmoplantar pustulosis: do we need to change? $\mathrm{Br} \mathrm{J}$ Dermatol. 2011;164:942-6.

6. Eriksson MO, Hagforsen E, Lundin IP, Michaelsson 
G. Palmoplantar pustulosis: a clinical and immunohistological study. Br J Dermatol. 1998;138:390-8.

7. Kasche A, Pfab F, Hein R, Athanasiadis GI, Ollert M, Ring J, et al. Severe psoriasis pustulosa palmaris et plantaris (Barber-Königsbeck) treated successfully with soluble tumour necrosis factor receptor fusion protein (etanercept). J Eur Acad Dermatol Venereol. 2007;21:255-7.

8. Rallis E, Korfitis C, Stavropoulou E, Papaconstantis M. Onset of palmoplantar pustular psoriasis while on adalimumab for psoriatic arthritis: a "class effect" of TNF-alpha antagonists or simply an anti-psoriatic treatment adverse reaction? J Dermatol Treat. 2010;21:3-5.

9. Sanchez APG. Imunopatogênese da psoríase. An Bras Dermatol. 2010;85(5):747-9.

10. Smith $\mathrm{CH}$, Anstey AV, Barker JN, Burden AD, Chalmers RJ, Chandler DA, et al. British Association of Dermatologists' guidelines for biologic interventions for psoriasis 2009. Br J Dermatol. 2009;161:1019.

11. Moustou AE, Matekovits A, Dessinioti C, Antoniou C, Sfikakis PP, Stratigos AJ. Cutaneous side effects of anti-tumor necrosis factor biologic therapy: a clinical review. J Am Acad Dermatol. 2009;61:486-504.

12. Bissonnette R, Poulin $\mathrm{Y}$, Bolduc $\mathrm{C}$, Maari $\mathrm{C}$, Provost $\mathrm{N}$, Syrotuik J, et al. Etanercept in the treatment of palmoplantar pustulosis. J Drugs Dermatol. 2008;7:940-6.

13. Weinberg JM. Successful treatment of recalcitrant palmoplantar psoriasis with etanercept. Cutis. 2003;72:396-8.

14. Gottlieb AB, Matheson RT, Lowe N, et al. A randomized trial of etanercept as monotherapy for psoriasis. Arch Dermatol. 2003;139:1627-32.

15. Yawalkar N, Hunger RE. Successful treatment of recalcitrant palmoplantar pustular psoriasis with sequencial use of infl iximab and adalimumab. Dermatology. 2009;21:79-83.
16. Martins GA, Arruda L, Mugnaini ASB. Validação de questionários de avaliação da qualidade de vida em pacientes de psoríase. An Bras Dermatol. 2004;79(5):521-35.

17. Kimball $A B$, Gieler $U$, Linder D, Sampogna F, Warren RB, Augustin M. Psoriasis: is the impairment to a patient's life cumulative? JEADV. 2010;24(9):9891004.

18. Lima XT, Seidler EM, Lima HC, Kimball AB. Long-term safety of biologics in dermatology. Dermatol Ther. 2009;22:2-21.

19. Warren RB, Brown BC, Grindlay DJC, Griffiths CEM. What's new in psoriasis? Analysis of the clinical significance of new guidelines and systematic reviews on psoriasis published in 2008 and 2009. Clin Exp Dermatol. 2010;35:688-92.

20. Gordon KB, Gottlieb AB, Leonardi CL, Elewski $B E$, WANG $A$, Jahreis $A$, et al. Clinical response in psoriasis patients discontinued from and then reinitiated on etanercept therapy. J Dermatol Treat. 2006;17:9-17.

21. Moore A, Gordon KB, Kang S, Gottlieb AB, Freundlich $B$, Xia $H A$, et al. A randomized, open-label trial of continuous versus interrupted etanercept therapy in the treatmentof psoriasis. J Am Acad Dermatol. 2007;56:598-603.

22. Ortonne JP, Griffiths C, Daudén E, Strohal R, Robertson D, Pedersen R, et al. Efficacy and safety of continuous versus paused etanercept treatment in patients with moderate-to severe psoriasis over 54 weeks: the CRYSTEL study. Expert Rev Dermatol. 2008;3(6):657-65.

23. Barrera MV, Habicheyn S, Mendiola MV, Ceballos $\mathrm{EH}$. Etanercept in the treatment and retreatment of psoriasis in daily clinical practice. Eur $\mathrm{J}$ Dermatol. 2008;18(6):683-7.

Artigo recebido em: 15/06/2011

Artigo aceito em: 16/08/2011 